\title{
Tumor necrosis factor-related apoptosis inducing ligand overexpression and Taxol treatment suppresses the growth of cervical cancer cells in vitro and in vivo
}

\author{
XIAOJIE SUN ${ }^{1}$, MANHUA CUI $^{2}$, DING WANG ${ }^{3}$, BAOFENG GUO $^{1}$ and LING ZHANG ${ }^{3}$ \\ ${ }^{1}$ Department of Plastic Surgery, China-Japan Union Hospital of Jilin University, Changchun, Jilin 130033; \\ ${ }^{2}$ Department of Gynaecology and Obstetrics, The Second Hospital of Jilin University, Changchun, Jilin 130022; \\ ${ }^{3}$ Department of Pathophysiology, College of Basic Medical Science, Jilin University, Changchun, Jilin 130021 P.R. China
}

Received May 23, 2017; Accepted January 17, 2018

DOI: $10.3892 / \mathrm{ol} .2018 .8071$

\begin{abstract}
Tumor necrosis factor-related apoptosis inducing ligand (TRAIL) is a member of tumor necrosis factor (TNF) superfamily and functions to promote apoptosis by binding to cell surface death receptor (DR)4 and DR5. Cancer cells are more sensitive than normal cells to TRAIL-induced apoptosis, and TRAIL-based therapeutic strategies have shown promise for the treatment of cancer. The present study investigated whether enforced overexpression of TRAIL in cervical cancer cells promoted cell death in the presence or absence of Taxol, an important first-line cancer chemotherapeutic drug. Hela human cervical cancer cells were transfected with a TRAIL expression plasmid, and the effects of the combination treatment with Taxol on apoptosis was investigated in vitro and in tumor xenografts in vivo. The results indicated that Taxol treatment and TRAIL overexpression enhanced apoptosis compared with either treatment alone. The present data indicate that Taxol may enhance the pro-apoptotic effects of TRAIL overexpression in HeLa cells by increasing cleaved caspase-3 and DR5 expression levels and decreasing Bcl-2 expression levels. Furthermore, the findings suggest a possible novel treatment option for cervical cancer and uncovers a potential mechanism of the enhancing effects of Taxol on TRAIL-induced apoptosis.
\end{abstract}

Correspondence to: Dr Baofeng Guo, Department of Plastic Surgery, China-Japan Union Hospital of Jilin University, 126 Sendai Street, Changchun, Jilin 130033, P.R. China

E-mail: gbf_cc@sohu.com

Professor Ling Zhang, Department of Pathophysiology, College of Basic Medical Science, Jilin University, 126 Xinmin Street, Changchun, Jilin 130021, P.R. China

E-mail: zhangling3@jlu.edu.cn

Key words: tumor necrosis factor-related apoptosis inducing ligand, Taxol, cervical cancer, apoptosis, additive effect

\section{Introduction}

Cervical cancer is a common malignancy with an estimated 485000 new cases and 236000 deaths annually worldwide (1). Nearly all cases of cervical cancer are caused by human papillomavirus infection (2). Advances in novel diagnostic and therapeutic technologies have led to a considerable decline in cervical cancer morbidity and mortality over the last decade $(3,4)$. Current treatments for cervical cancer are surgery, radiotherapy and chemotherapy (5); however, additional therapies will be required to increase survival rates and reduce the need for surgery.

TNF-related apoptosis-inducing ligand (TRAIL) is a member of the tumor necrosis factor (TNF) superfamily and was originally cloned from human myocardial cell. TRAIL can bind to five receptors, of which two, DR4 (TRAILR1) and DR5 (TRAILR2), induce apoptosis. Upon TRAIL binding, FAS-associated protein with death domain (FADD) and caspase 8 sequentially recruited to the DR4/DR5 intracellular domain, resulting in formation of a death-inducing signaling complex (DISC) (6). Downstream effector molecules, including caspase-3, are then activated and induce the biochemical and morphological hallmarks of apoptosis via cleavage of numerous cellular proteins (6-8). At present, several types of cancers have been reported to be sensitive to TRAIL-induced apoptosis in vitro and in vivo (6,9-11). In addition, radiotherapy and chemotherapy are known to enhance the effects of TRAIL by inducing the DR4 or DR5 expression (12-16).

Taxol, an important first-line drug in cervical cancer therapy, was first isolated from Taxus brevifolia in 1962 (17). Taxol inhibits the disassembly of microtubule polymers, which causes the arrest of cancer cells in metaphase and leads to apoptosis (17). Taxol is a wide-spectrum anti-tumor drug and is used to treat many cancers, including lung cancer, ovarian cancer (18-20). However, Taxol has weak anti-tumor effects and must be used in combination with cisplatin or other chemotherapies (19).

Two major apoptosis signaling pathways exist in mammalian cells: The intrinsic pathway, which is controlled by the Bcl-2 family of proteins and is induced by chemotherapy and radiotherapy, and the extrinsic pathway, which is mediated by 
the TNF receptor superfamily $(6,21)$. In cancer cells, intrinsic pathway-induced death can be enhanced by concomitant activation of the extrinsic pathway; thus, inhibition of both pathways could be a powerful method of inducing apoptosis in cancer cells (6). To test this, we constructed a recombinant plasmid to overexpress human $\mathrm{TRAIL}_{114-281}$ protein in the human cervical carcinoma cell line, HeLa. We examined the effects of TRAIL overexpression in combination with Taxol treatment on cell growth and apoptosis in vitro and in vivo, and investigated their possible underlying mechanisms of action.

\section{Materials and methods}

Plasmids construction, cell culture and transfection. To generate a TRAIL overexpression plasmid, we cloned the human TRAIL functional domain (amino acids 144-281; GeneBank accession No. NM_003810), and the sequence was cloned into a plasmid encoding GFP and ampicillin- and neosporin-resistance genes. The TRAIL sequence was amplified by PCR with the following primers: P1, 5'-CCGAGATCTGTGAGAGAAGAGGTCC-3'; P2, 5'-CTTGTCGACTTAGCCAACTAAAAAGGCCC-3'. A signal peptide sequence was placed in front of TRAIL to ensure passage of the translated protein through cell membranes into the extracellular medium for interaction with receptors. The of signal peptide primers were: P1: 5'-CTAGCATGGCCCTGTGGATGCGCC TCCTGCCCCTGCTGGCGCTGCTGGCCCTCTGGGGAC CTGACCCAGCCGCAGCC-3'; P2, 5'-CTAGGGCTGCGG CTGGGTCAGGTCCCCAGAGGGCCAGCAGCGCCAGCA GGGGCAGGAGGCGCATCCACAGGGCCATG-3'. The human cervical carcinoma cell line HeLa was obtained from the Shanghai Institute of Cell Biology, Chinese Academy of Sciences (Shanghai, China). Cells were cultured in IMDM containing 10\% fetal bovine serum and were maintained at $37^{\circ} \mathrm{C}$ in a $5 \% \mathrm{CO}_{2}$ atmosphere. The cells were transfected with plasmids pTRAIL or pVector (empty vector control) using Lipofectamine 2000 (Invitrogen; Thermo Fisher Scientific, Inc., Waltham, MA, USA). G418 at $800 \mu \mathrm{g} / \mu \mathrm{l}$ was added to the medium to select for stable HeLa-TRAIL and HeLa-vect cell lines.

Western blot analysis. Cell lysis protein quantification, and Western blotting analysis were carried out as described previously (22). Antibodies against $\beta$-actin, Bcl-2, cleaved caspase-3, and TRAIL were purchased from Wuhan Boster Biological Technology, Ltd. (Wuhan, China).

Cell proliferation, cell cycle and apoptosis analysis. Cell proliferation was assayed using a 3-(4,5-dimethylthiazol-2-yl)-2,5-diphenyltetrazolium bromide(MTT) kit (Sigma, USA) according to the manufacturer's instructions. Inhibition of the cell growth rate was calculated as \% inhibition $=(1-$ absorbance of the experimental group/absorbance of the control group) $\times 100 \%$. Cell cycle phase and apoptosis ware determined by flow cytometry. In brief, stably transfected HeLa lines were resuspended in phosphate-buffered saline, and DNA was stained by the addtion $50 \mu \mathrm{g} / \mathrm{ml}$ propidium iodide (PI) (Beckman Coulter, USA). The percentage of apoptotic cells and cells in different cell cycle phases were quantified using an Epics-XL-MCL flow cytometer (Beckman Coulter). HeLa cell apoptosis was also analyzed by staining with acridine orange/ethidium bromide
(AO/EB) followed by fluorescence microscopy. Cell death in excised tumor xenografts was analyzed by hematoxylin and eosin (H\&E) and terminal deoxynucleotidyl transferase dUTP nick-end labeling (TUNEL) staining as described previously $(22,23)$. To quantify cell death in tissue sections, we randomly selected five views for each group and analyzed TUNEL-positive cells using an HPIAS-100 image analysis system.

Animal care. Female Nude mice were purchased from the Beijing Institute for Experimental Animals. All animals were cared for in compliance with institutional guidelines and ethical approval was received from the Animal Experimental Ethics Committee of Jilin University.

Tumor xenografts model. Four groups of female nude mice $(n=5)$ were injected subcutaneously (s.c.) in the right flank with $2 \times 10^{6} \mathrm{HeLa}$ cells, and the tumor were allowed to grow until they reached to $7 \mathrm{~mm}$ in diameter. After the point, the four groups of mice were randomly assigned to receive injection of liposomes mixed with pVector or pTRAIL plasmids (150 $\mu \mathrm{g}$ per mouse) and co-injections of vehicle or Taxol (20 mg/kg) once a day for 3 weeks respectively. The mice were then sacrificed and tumors were excised and evaluated by Immunohistochemistry, H\&E and TUNEL staining.

Immunohistochemical staining. Excised tumor samples were prepared for immunohistochemical detection of cleaved caspase- 3 and Bcl-2 protein using a streptavidin-biotin-peroxidase complex staining kit (SABC; Wuhan Boster Biological Technology, Ltd.). Antibody to cleaved caspase-3 and Bcl-2 were purchased from Wuhan Boster Biological Technology, Ltd. To quantify staining, 5 fields of view were randomly selected for each experimental group and cells were analyzed using the HPIAS-100 image analysis system.

Semi-quantitation RT-PCR. Total RNA was isolated from cells using TRIzol reagent (Ambion; Thermo Fisher Scientific, Inc.) according to the manufacturer's protocol. cDNA was generated by reverse transcription of $2 \mu \mathrm{g}$ samples of total RNA using a RevertAid First Strand cDNA Synthesis kit (Thermo Fisher Scientific, Inc.). The PCR primers were: $\beta$-actin sense: 5'CTG GGACGACATGGAGAAAA-3' and antisense: 5'-AAGGAA GGCTGGAAGAGTGC-3'; DR4 sense 5'-CTGAGCAACGCA GACTCGCTGTCCAC-3' and antisense 5'-TCCAAGGACACG GCAGAGCCTGTGCCAT-3'; and DR5 sense 5'-GCCTCATGG ACAATGAGATAAAGGTGGCT-3'and antisense 5'-CCAAAT CTCAAAGTACGCACAAACGG-3'. The PCR reaction conditions were as follows: denaturation at $94^{\circ} \mathrm{C}$ for $3 \mathrm{~min}$ followed by 30 amplification cycles $\left(94^{\circ} \mathrm{C}\right.$ for $30 \mathrm{sec}, 59^{\circ} \mathrm{C}$ for $30 \mathrm{sec}$, $72^{\circ} \mathrm{C}$ for $30 \mathrm{sec}$ ) and a final extension of $72^{\circ} \mathrm{C}$ for $5 \mathrm{~min}$. The PCR products were analyzed by $1 \%$ agarose gel electrophoresis containing $5 \mu \mathrm{g} / \mathrm{ml} \mathrm{EB}$, and the bands were visualized using a GIS Gelatum imaging system (Tanon Science and Technology Co., Ltd., Shanghai, China).

Statistical analysis. The data are expressed as mean values \pm standard deviation (SD). For both in vitro and in vivo experiments, group differences were analyzed by 
A



HeLa

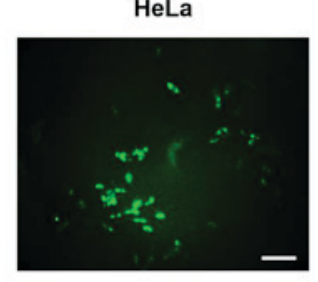

HeLa-TRAIL
B

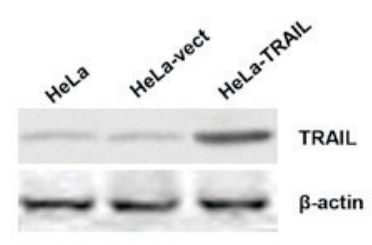

C

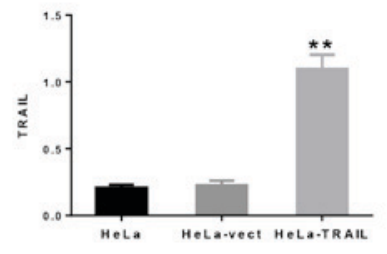

Figure 1. TRAIL protein expression in HeLa cells transfected with pVector or pTRAIL. (A) Expression of GFP in HeLa cells stably expressing TRAIL vs. uninfected cells. Scale bar, $50 \mu \mathrm{m}$. (B) Western blot analysis of TRAIL expression in HeLa, HeLa-vect, and HeLa-TRAIL cells. (C) Quantification of TRAIL protein levels from three separate experiments. Data are presented as the means $\pm \mathrm{SD} .{ }^{* *} \mathrm{P}<0.01$ vs. HeLa or HeLa-vect cells.

Kruskal-Wallis test followed by Dunn's multiple comparisons test. All experiments were repeated at least three times. Statistical calculations were performed using SigmaStat software (SPSS v20; SPSS, Inc., Chicago, IL, USA). P<0.05 was considered to indicate a statistically significant difference.

\section{Results}

Construction of a human TRAIL overexpression plasmids. We choose the functional domain (amino acids 144-281) of human TRAIL by constructing a plasmid (pTRAIL) that also encoded the GFP gene and a signal peptide preceding TRAIL to ensure passage of the protein through membranes to the extracellular medium. HeLa cells were transfected with the empty vector (control, pVector) or pTRAIL, and stable transfectants were selected by growth in G418. The transfection efficiency was virtually identical for both cell types, as detected by the expression of GFP (Fig. 1A). As expected, expression of TRAIL was significantly higher in HeLa cells transfected with pTRAIL (HeLa-TRAIL) compared with cells transfected with the empty vector (HeLa-vect) or untransfected HeLa cells (Figs. 1B and C).

\section{Effects of combination treatment with TRAIL and Taxol}

Suppression of HeLa cell proliferation. We first examined the growth of f HeLa, HeLa-vect, and HeLa-TRAIL cells, and found that pTRAIL-transfected cells showed reduced proliferation after $24 \mathrm{~h}$ compared to HeLa-vect and uninfected Hela cells (Fig. 2A). We next determined whether Taxol treatment could act in an additive manner with TRAIL transfection to suppress HeLa cells survival. We found that Taxol dose-dependently inhibited the survival of HeLa cells, and fewer HeLa-TRAIL cells than HeLa-vect or HeLa cells were alive after $24 \mathrm{~h}$ incubation in the presence of $\geq 10 \mu \mathrm{g} / \mathrm{ml}$ Taxol (Fig. 2B). Moreover, Hela-TRAIL cells treated with a constant Taxol concentration (40 ug/ml) showed a decrease in survival over time (Fig. 2C).

Effects of TRAIL and Taxol on apoptosis. To determine whether TRAIL and Taxol suppress HeLa cell growth and survival by inducing apoptosis, we used two assays: Flow cytometry of PI-stained cells and fluorescence microscopy of AO/EB-stained cells. Flow cytometry analysis showed that treatment of HeLa-vect cells with Taxol or transfection with pTRAIL increased the percentage of apoptotic cells to a similar extent (Table I). However, apoptosis was further increased by treatment of HeLa-TRAIL cells with Taxol (Table I), indicating an additive effect. In the second approach, cells were stained with $\mathrm{AO} / \mathrm{EB}$ and analyzed by fluorescence microscopy. This dual staining method canc identify early $(\mathrm{AO}+)$ and late $(\mathrm{AO}+\mathrm{EB}+)$ apoptotic cells. HeLa-TRAIL, Hela-vect + Taxol and HeLa-TRAIL + Taxol cultures all showed evidence of apoptosis, whereas HeLa-vect cells did not (Fig. 3A). More apoptotic cells were detected in the HeLa-TRAIL + Taxol group compared with either the HeLa-TRAIL or HeLa-vect + Taxol groups, indicating that TRAIL and Taxol induce both early and late apoptosis in HeLa cells. These data suggest that TRAIL overexpression and Taxol treatment in combination induced HeLa cell apoptosis more efficiently than either one alone.

Expression of apoptosis-related proteins in HeLa cells. To determine the mechanism by which TRAIL and Taxol induce Hela cell apoptosis, we examined the expression of two apoptosis-associated proteins; pro-apoptotic cleaved caspase-3 and anti-apoptotic Bcl-2 (Fig. 3B and C). The highest expression of cleaved caspase- 3 was observed in Taxol-treated HeLa-TRAIL cells, whereas only the groups treated with Taxol showed a decrease in Bcl-2 levels. These finding suggest that Taxol, but not TRAIL, modulates Bcl-2 expression during HeLa cell apoptosis. In contrast, cleaved caspase-3 appears to contribute to apoptosis induced by both TRAIL and Taxol. We next examined the mRNA expression level of DR4 and DR5, two apoptosis-related TRAIL receptors, in HeLa cells treated with different concentrations of Taxol. As shown in Fig. 3D, Taxol caused a dose-dependent increase in DR5 mRNA levels but had no effect on DR4 mRNA (data not shown). Thus, Taxol may enhance the pro-apoptosis function of TRAIL by increasing the expression of DR5, but not DR4 in HeLa cells.

Antitumor activity of TRAIL and Taxol in vivo. To evaluate the effects of the combinative treatment of TRAIL and Taxol on cervical cancer growth in vivo, we used a nude mouse tumor xenograft model. Hela cells were inoculated s.c. into female nude mice, and tumors allowed to grow to a diameter of $7 \mathrm{~mm}$. The groups were then randomly assigned to receive injections of pVector or pTRAIL plasmid and either vehicle or Taxol for 3 weeks. Animals were then sacrificed and the tumors were excised for analysis.

The tumor volumes and weights and are shown in Fig. 4A-C. Tumors from mice treated with pTRAIL and/or Taxol were significantly diminished compared with tumors from mice receiving vehicle (saline) or pVector treatments. As shown in Table II, there were no significant difference in average mouse weights between groups $(\mathrm{P}>0.05)$. However, tumors from mice treated with pTRAIL + Taxol had markedly reduced weight and volume compared with tumors from the other three mouse groups. 
Table I. Effect of TRAIL overexpression and Taxol treatment on HeLa cell cycle phase and apoptosis.

\begin{tabular}{lccr}
\hline Group $(\mathrm{n}=5)$ & Apoptotic cells $(\%$, mean $\pm \mathrm{SD})$ & G2-M $(\%$, mean $\pm \mathrm{SD})$ & $\mathrm{S}(\%$, mean $\pm \mathrm{SD})$ \\
\hline Hela-vect & $6.8 \pm 0.65$ & $2.8 \pm 1.83$ & $20.6 \pm 2.17$ \\
Hela-vect+Taxol & $17.5 \pm 2.32^{\mathrm{a}}$ & $35.7 \pm 2.96^{\mathrm{a}}$ & $4.4 \pm 0.94^{\mathrm{a}}$ \\
Hela-TRAIL & $18.6 \pm 3.66^{\mathrm{a}}$ & $13.5 \pm 2.46^{\mathrm{a}}$ & $38.3 \pm 3.87^{\mathrm{a}}$ \\
Hela-TRAIL + Taxol & $42.3 \pm 6.21^{\mathrm{a}-\mathrm{c}}$ & $32.1 \pm 2.53^{\mathrm{a}, \mathrm{c}}$ & $17.2 \pm 4.38^{\mathrm{b}, \mathrm{c}}$ \\
\hline
\end{tabular}

${ }^{\mathrm{a}} \mathrm{P}<0.01$ vs. HeLa-vect; ${ }^{\mathrm{P}} \mathrm{P}<0.01$ vs. HeLa-vect + Taxol; ${ }^{\mathrm{c}} \mathrm{P}<0.01$ vs. HeLa-TRAIL + Taxol. TRAIL, tumor necrosis factor-related apoptosis inducing ligand; vect, vector; SD, standard deviation.


Figure 2. Suppression of HeLa cells transfected with pVector or pTRAIL and treated with Taxol. (A) MTT assay of inhibition of cell proliferation by Taxol. (B, C) Survival of HeLa cells incubated with varying concentrations of Taxol for $24 \mathrm{~h}$ (B) or Taxol at $40 \mu \mathrm{g} / \mathrm{ml}$ for the indicated times (C). Data are presented as the means $\pm \mathrm{SD}$ of $\mathrm{n} \geq 3$.

The excised tumors were sectioned and examined by H\&E staining and TUNEL staining to detect fragmented DNA (Fig. 5A, B and E). Tumors from the mouse groups treated with pTRAIL, Taxol, or pTRAIL + Taxol showed elevated levels of apoptotic cells compared with the pVector group, with the pTRAIL + Taxol group showing the highest levels. These tumor sections were characterized by increased nuclear condensation and DNA fragmentation (indicated by TUNEL-positive cells), whereas tumor sections from the pVector group had granular cytoplasm and no TUNEL-positive cells. These data demonstrated that the combination of pTRAIL and Taxol exerts a stronger anti-tumor pro-apoptotic effect in vivo compared with either treatment alone.

Finally, we examined the expression of cleaved caspase-3 and Bcle-2 protein in xenograft tumors by immunohistochemical staining (Fig. 5C and D). Consistent with the TUNEL staining results, tumors from mice treated with pTRAIL and/or Taxol showed increased cleaved caspase-3 and decreased Bcl-2 expression compared with tumors from HeLa-vect and/or saline-treated mice. Quantification of protein staining showed that expression was highest in the tumors from pTRAIL + Taxol-treated mice (Fig. 5F).

\section{Discussion}

Deaths from uterine cancer declined by more than $80 \%$ between 1930 and 2012, largely due to the introduction of the Papanicolaou test for early cervical cancer, which is predominantly caused by human papillomavirus infection (4). However, cervical cancer is still the third most common carcinoma among women worldwide and results in 236000 deaths each year (1); thus there is a pressing need for new and/or improved treatments.
TRAIL, a member of the TNF protein superfamily, induces apoptosis by binding to the cell surface death receptors DR4 and DR5 (6). TRAIL-induced apoptosis has been shown to be enhanced by combination treatment with radiotherapy or chemotherapy $(12,13)$. Taxol, a classical chemotherapeutic agent, exerts its anti-tumor effect by arresting cells in metaphase, which induces apoptosis. Cisplatin and Taxol both show synergistic effects with TRAIL in non-small cell lung cancer (24). There are also reports that the combination of TRAIL and Taxol enhances apoptosis in gastric cancer and hepatocellular carcinoma cells $(25,26)$. Here, we examined the efficacy of TRAIL and Taxol combination therapy in cervical cancer cells in vitro and in vivo.

To study the anti-tumor effect of TRAIL, we transfected HeLa cells with a plasmid encoding a signal sequence and the functional domain of TRAIL, resulting in constitutive protein production and secretion. As expected, Hela cells overexpressing TRAIL showed poorer proliferation than normal Hela cells or the pVector control cells. We also showed that Taxol could enhance the damaging effect of high TRAIL expression and significantly decreased the survival of HeLa-TRAIL cells in a dose- and time dependent manner. Since the combination of TRAIL and Taxol had previously been shown to enhance apoptosis in non-small cell lung cancer cells (24), we also investigated their effects on apoptosis in HeLa cells. Indeed, microscopic and flow cytometric analysis identified a significantly higher degree of apoptosis in the pTRAIL + Taxol group compared with the pTRAIL or Taxol groups. To determine the mechanism of enhanced apoptosis, we measured cellular levels of cleaved caspase- 3 and Bcl-2 as markers of extrinsic and intrinsic apoptosis, respectively. As expected, the expression of cleaved caspase- 3 was consistent with the degree of apoptosis induced by pTRAIL and/or Taxol. However, Bcl-2, a 


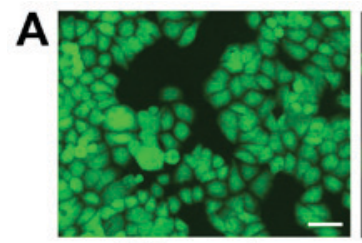

HeLa-vect

B

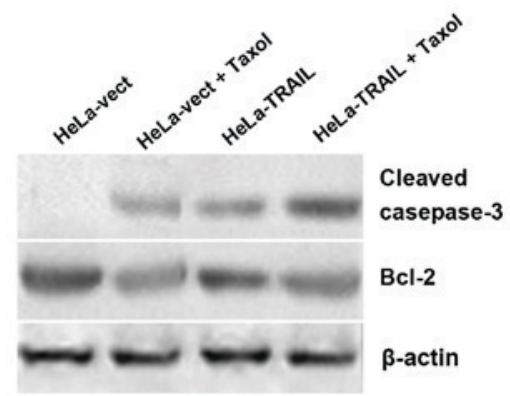

D

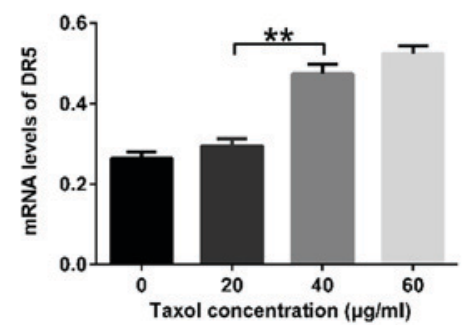

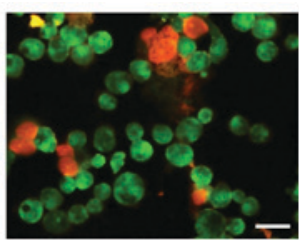

HeLa-vect + Taxol

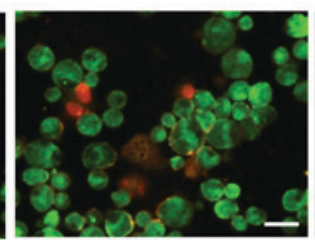

HeLa-TRAIL

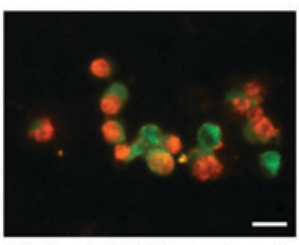

HeLa-TRAIL + Taxol



Figure 3. Apoptosis of HeLa-vect and HeLa-TRAIL cells treated with Taxol. (A) AO/EB-stained cells. Scale bar, $100 \mu \mathrm{m}$. (B) Western blot analysis of cleaved caspase-3 and Bcl-2 protein expression. (C) Quantification of cleaved caspase-3 and Bcl-2 protein levels from three experiments. (D) DR5 mRNA levels in HeLa cells treated with different concentrations of Taxol. Data are from three separate experiments. Data are presented as the means $\pm \mathrm{SD}$. ${ }^{* *} \mathrm{P}<0.01$.

A

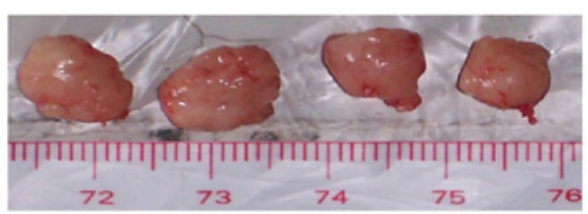

Empty vector

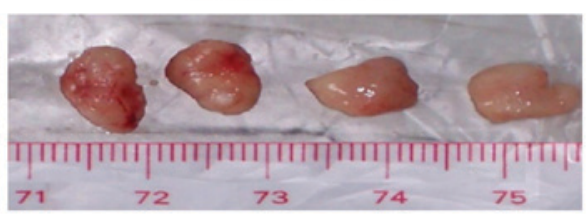

Taxol

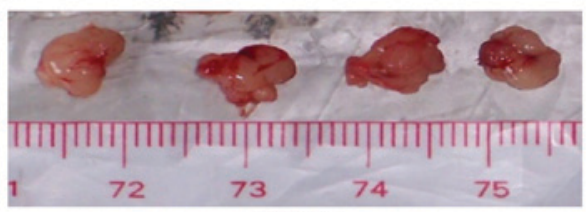

PTRAIL

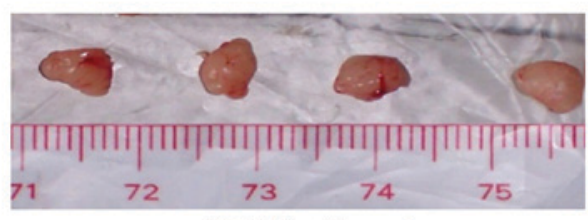

pTRAIL +Taxol
B

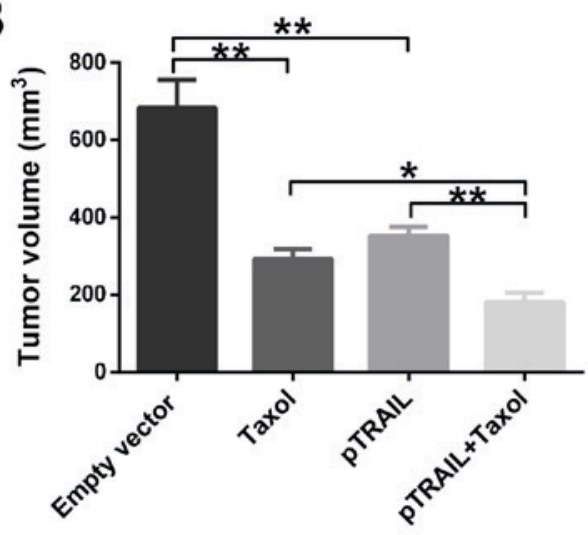

C

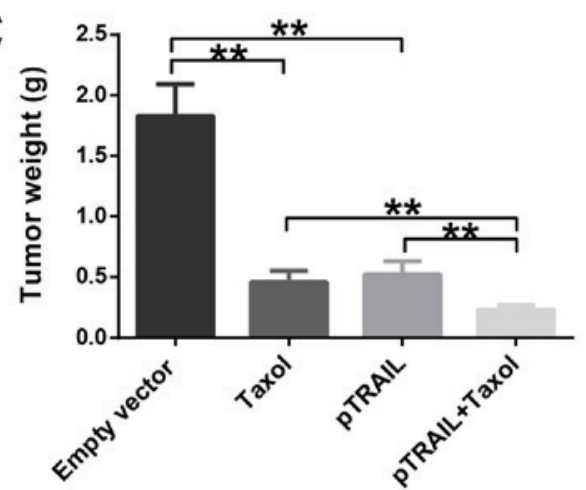

Figure 4. Inhibition of HeLa tumor xenograft growth by expression of pTRAIL and/or treatment with Taxol. (A) Images of tumors excised from mice treated with different regimens for 21 days. (B, C) Volume (B) and weight $(\mathrm{C})$ of tumors after treatment for 21 days (n=5). Data are presented as the means $\pm \mathrm{SD}$. ${ }^{*} \mathrm{P}<0.05,{ }^{* *} \mathrm{P}<0.01$. 
Table II. Analysis of tumors and mice bearing HeLa tumor xenografts.

\begin{tabular}{lccc}
\hline Group $(\mathrm{n}=4)$ & Mean weight of the nude mice $(\mathrm{g})$ & Mean weight of tumor $(\mathrm{g})$ & Mean volume of tumor $\left(\mathrm{mm}^{3}\right)$ \\
\hline Empty vector & $25.21 \pm 2.94$ & $1.98 \pm 0.35$ & $702.75 \pm 117.63$ \\
Taxol & $24.18 \pm 2.23$ & $0.46 \pm 0.09^{\mathrm{a}}$ & $292.68 \pm 25.30^{\mathrm{a}}$ \\
pTRAIL & $24.34 \pm 2.76$ & $0.52 \pm 0.11^{\mathrm{a}}$ & $353.14 \pm 22.39^{\mathrm{a}}$ \\
pTRAIL+Taxol & $25.23 \pm 2.71$ & $0.23 \pm 0.04^{\mathrm{a}-\mathrm{c}}$ & $181.31 \pm 24.57^{\mathrm{a}, \mathrm{c}}$ \\
\hline
\end{tabular}

${ }^{\mathrm{a}} \mathrm{P}<0.01$ vs. Empty vector; ${ }^{\mathrm{b}} \mathrm{P}<0.01 \mathrm{vs}$. Taxol; ${ }^{\mathrm{c}} \mathrm{P}<0.01$ vs. pTRAIL. pTRAIL, plasmid tumor necrosis factor-related apoptosis inducing ligand.

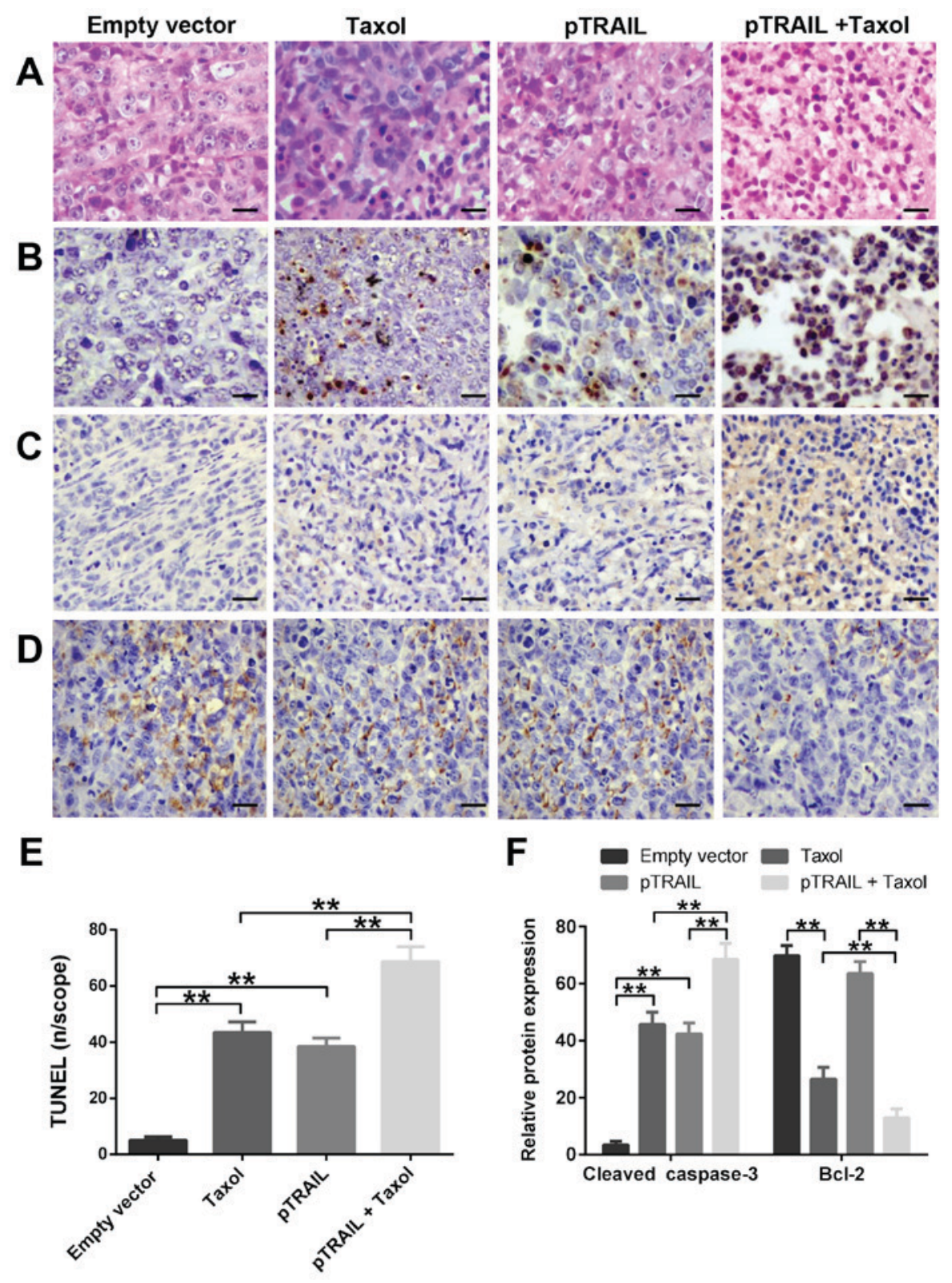

Figure 5. H\&E, TUNEL, and immunohistochemical staining of HeLa tumor xenografts. (A, B) H\&E and TUNEL staining. (C, D) Immunohistochemical staining of cleaved caspase-3 (C) and Bcl-2 (D). Scale bar, $100 \mu \mathrm{m}$ ). (E) Quantification of the percentage of apoptotic cells in the TUNEL assay. (F) Quantification of cleaved caspase-3 and Bcl-2 protein expression detected by immunohistochemistry. Data are presented as the means $\pm \mathrm{SD}$. ${ }^{* *} \mathrm{P}<0.01$.

hallmark anti-apoptotic protein, was only downregulated after treatment with Taxol. TRAIL has been reported to synergize with luteolin, a naturally occurring flavonoid that upregulates DR5, to induce apoptosis in HeLa cell (26). In the present study, we found that DR5 expression in HeLa cells increased in parallel with Taxol concentration, indicating that Taxol may enhance the pro-apoptotic function of TRAIL by increasing DR5 expression. 
Using a mouse tumor xenograft model, we showed that the volumes and weights of HeLa-derived tumors were decreased significantly by combined overexpression of TRAIL and treatment with Taxol compared with either treatment alone. We evaluated the tumor sections by H\&E and TUNEL staining and found that the cell morphology and degree of DNA fragmentation were in accordance with the in vitro findings. Cleaved caspase-3 protein expression was elevated in TRAIL-transfected and Taxol-treated tumors compared with either treatment alone. Similarly, tumors from the pTRAIL + Taxol group expressed lower Bcl-2 levels than tumors from mice treated with Taxol alone, confirming that TRAIL enhances the Taxol-induced intrinsic apoptotic pathway in vivo. However, the mechanism by which this occurs remains unclear.

In this study, we found that the combination of TRAIL overexpression and Taxol treatment significantly increased apoptosis of a human cervical carcinoma cell line compared with either TRAIL overexpression or Taxol treatment alone. Moreover, this additive effect may derive from Taxol-induced upregulation of DR5, a TRAIL receptor that connects to the downstream extrinsic apoptosis pathway.

A number of different vectors are currently being used for gene therapy of malignant tumors. Whereas early vectors were non-specific, novel vectors, especially viruses, have been developed that target cancer cells but leave normal cells unharmed(27). We have reported that attenuated Salmonella typhimurium has potential utility for transgene delivery, since it shows $>1,000$-fold preferential accumulation in tumors compared with normal tissues (28). Our results suggest that this delivery system might be useful for combination therapy with TRAIL plasmids and Taxol for the treatment of cancer.

\section{Acknowledgements}

The present study was funded by the National Natural Science Foundation of China (grant nos. 81472344 and 81773217) and Jilin Provincial Education Department (grant no. Jijiaokehezi [2016]455) and the International Cooperation Project of Jilin Provincial Science and Technology Department (grant no. 20150414031GH) and Jilin University Bethune Plan B Projects (No. 2015220). The authors would like to thank Dr Anne M. O'Rourke for editing the English text of a draft of this manuscript.

\section{References}

1. Global Burden of Disease Cancer Collaboration, Fitzmaurice C Dicker D, Pain A, Hamavid H, Moradi-Lakeh M, MacIntyre MF Allen C, Hansen G, Woodbrook R, et al: The global burden of cancer 2013. JAMA Oncol 1: 505-527, 2015.

2. Crosbie EJ, Einstein MH,Franceschi S and Kitchener HC: Human papillomavirus and cervical cancer. Lancet 382: 889-899, 2013.

3. Jemal A, Siegel R, Ward E, Hao Y, Xu J, Murray T and Thun MJ: Cancer statistics, 2008. CA Cancer J Clin 58: 71-96, 2008.

4. Siegel RL, Miller KD and Jemal A: Cancer statistics, 2016. CA Cancer J Clin 66: 7-30, 2016.

5. Yee GP, de Souza P and Khachigian LM: Current and potential treatments for cervical cancer. Curr Cancer Drug Targets 13: 205-220, 2013

6. Johnstone RW, Frew AJ and Smyth MJ: The TRAIL apoptotic pathway in cancer onset, progression and therapy. Nat Rev Cancer 8: 782-798, 2008.

7. Ashkenazi A and Dixit VM: Apoptosis control by death and decoy receptors. Curr Opin Cell Biol 11: 255-260, 1999.

8. Falschlehner C, Emmerich CH, Gerlach B and Walczak H: TRAIL signalling: Decisions between life and death. Int $\mathbf{J}$ Biochem Cell Biol 39: 1462-1475, 2007.
9. Horak P, Pils D, Kaider A, Pinter A, Elandt K, Sax C, Zielinski CC, Horvat R, Zeillinger R, Reinthaller A and Krainer M: Perturbation of the tumor necrosis factor-related apoptosis-inducing ligand cascade in ovarian cancer: Overexpression of FLIPL and deregulation of the functional receptors DR4 and DR5. Clin Cancer Res 11: 8585-8591, 2005.

10. Martinez-Ferrandis JI, Rodríguez-López R, Milne RL, González E, Cebolla E, Chirivella I, Zamora P, Arias JI, Palacios S, Cervantes A, et al: Polymorphisms in TRAIL receptor genes and risk of breast cancer in Spanish women. Cancer Biomark 3: 89-93, 2007.

11. Sanlioglu AD, Korcum AF, Pestereli E, Erdogan G, Karaveli S, Savas B, Griffith TS and Sanlioglu S: TRAIL death receptor-4 expression positively correlates with the tumor grade in breast cancer patients with invasive ductal carcinoma. Int J Radiat Oncol Biol Phys 69: 716-723, 2007.

12. Gliniak B and Le T: Tumor necrosis factor-related apoptosis-inducing ligand's antitumor activity in vivo is enhanced by the chemotherapeutic agent CPT-11. Cancer Res 59: 6153-6158, 1999.

13. Hori T, Kondo T, Kanamori M, Tabuchi Y, Ogawa R, Zhao QL, Ahmed K, Yasuda T, Seki S, Suzuki K and Kimura T: Ionizing radiation enhances tumor necrosis factor-related apoptosis-inducing ligand (TRAIL)-induced apoptosis through up-regulations of death receptor 4 (DR4) and death receptor 5 (DR5) in human osteosarcoma cells. J Orthop Res 28: 739-745, 2010.

14. Sussman RT, Ricci MS, Hart LS, Sun SY and El-Deiry WS: Chemotherapy-resistant side-population of colon cancer cells has a higher sensitivity to TRAIL than the non-SP, a higher expression of c-Myc and TRAIL-receptor DR4. Cancer Biol Ther 6: 1490-1495, 2007.

15. Finnberg NK, Gokare P, Navaraj A, Lang Kuhs KA, Cerniglia G, Yagita H, Takeda K, Motoyama N and El-Deiry WS: Agonists of the TRAIL death receptor DR5 sensitize intestinal stem cells to chemotherapy-induced cell death and trigger gastrointestinal toxicity. Cancer Res 76: 700-712, 2016.

16. Oliver PG, LoBuglio AF, Zhou T, Forero A, Kim H, Zinn KR, Zhai G, Li Y, Lee CH and Buchsbaum DJ: Effect of anti-DR 5 and chemotherapy on basal-like breast cancer. Breast Cancer Res Treat 133: 417-426, 2012.

17. Weaver BA: How Taxol/paclitaxel kills cancer cells. Mol Biol Cell 25: 2677-2681, 2014.

18. Ao X, Nie P, Wu B, Xu W, Zhang T, Wang S, Chang H and Zou Z: Decreased expression of microRNA-17 and microRNA-20b promotes breast cancer resistance to taxol therapy by upregulation of NCOA3. Cell Death Dis 7: e2463, 2016.

19. Li W, Wan L, Zhai LY and Wang J: Effects of SC-560 in combination with cisplatin or taxol on angiogenesis in human ovarian cancer xenografts. Int J Mol Sci 15: 19265-19280, 2014.

20. Peng C, Hao Y, Zhao Y, Sun Q, Zhao X and Cong B: Effect of Smac and Taxol on non-small-cell lung cancer. Acta Biochim Biophys Sin (Shanghai) 46: 387-393, 2014.

21. Johnstone RW, Ruefli AA and Lowe SW: Apoptosis: A link between cancer genetics and chemotherapy. Cell 108: 153-164, 2002.

22. Gao L, Zhang L, Hu J, Li F, Shao Y, Zhao D, Kalvakolanu DV, Kopecko DJ, Zhao X and Xu DQ: Down-regulation of signal transducer and activator of transcription 3 expression using vector-based small interfering RNAs suppresses growth of human prostate tumor in vivo. Clin Cancer Res 11: 6333-6341, 2005.

23. Li X, Zhang L, Shao Y, Liang Z, Shao C, Wang B, Guo B, Li N, Zhao X, Li Y and Xu D: Effects of a human plasma membrane-associated sialidase siRNA on prostate cancer invasion: Biochem Biophys Res Commun 416: 270-276, 2011.

24. Fan QL, Zou WY, Song LH and Wei W: Synergistic antitumor activity of TRAIL combined with chemotherapeutic agents in A549 cell lines in vitro and in vivo. Cancer Chemother Pharmacol 55: 189-196, 2005.

25. Chen L, Chen D, Gong M, Na M, Li L, Wu H, Jiang L, Qian Y, Fang $\mathrm{G}$ and Xue X: Concomitant use of Ad5/35 chimeric oncolytic adenovirus with TRAIL gene and taxol produces synergistic cytotoxicity in gastric cancer cells. Cancer Lett 284: 141-148, 2009.

26. Horinaka M, Yoshida T, Shiraishi T, Nakata S, Wakada M, Nakanishi R, Nishino H and Sakai T: The combination of TRAIL and luteolin enhances apoptosis in human cervical cancer HeLa cells. Biochem Biophys Res Commun 333: 833-838, 2005.

27. Pazarentzos E and Mazarakis ND: Anticancer gene transfer for cancer gene therapy. Adv Exp Med Biol 818: 255-280, 2014.

28. Zhang L, Gao L, Zhao L, Guo B, Ji K, Tian Y, Wang J, Yu H, Hu J, Kalvakolanu DV, et al: Intratumoral delivery and suppression of prostate tumor growth by attenuated Salmonella enterica serovar typhimurium carrying plasmid-based small interfering RNAs. Cancer Res 67: 5859-5864, 2007.

This work is licensed under a Creative Commons Attribution-NonCommercial-NoDerivatives 4.0 International (CC BY-NC-ND 4.0) License. 